\title{
Realidade Virtual e Ferramentas Cognitivas Usadas como Auxílio para o Ensino de Física
}

Luciano Ferreira Silva, PPGEE/UFU, DCC/UFRR, luciano@mat.ufu.br

Ezequiel Roberto Zorzal ${ }^{1}$, PPGEE/UFU, CEFET-SP, ezorzal@gmail.com

Mônica Rocha Ferreira de Oliveira, PPGEE/UFU, monica-rocha@ hotmail.com

Alexandre Cardoso, PPGEE/UFU, alexandre@ufu.br

Edgard A. Lamounier Jr. PPGEE/UFU, lamounier@ufu.br

Elise B. Mendes, FACED/UFU, elise@ufu.br

Eduardo Kojy Takahashi, INFIS/UFU, ektakahashi@ufu.br

Silvia Martins, INFIS/UFU, smartins@infis.ufu.br

Resumo: Este artigo descreve um sistema computacional elaborado para ser ferramenta de ensino de Física do Ensino Médio, com uso de técnicas de Realidade Virtual. Por meio de estratégias psico-pedagógicas, fundamentadas no paradigma de Mapas Conceituais, o sistema simula um laboratório virtual que permite ao estudante construir seu conhecimento através da criação e análise de experimentos físicos.

Palavras-chave: Realidade Virtual, Mapas Conceituais, Ferramentas Cognitivas.

\section{Virtual Reality and Cognitive Tools Used as Aid for Physics Teaching}

Abstract: This article describes a computer-based prototype system as a tool to support the teaching o Physics of the Medium Teaching, through Virtual Reality techniques. Using psico-pedagogic strategies, based upon the Conceptual Maps paradigm, the system simulates a virtual laboratory that allows the student to construct his own knowledge, through the creation and analysis of physical experiments.

Keywords: Virtual Reality, Conceptual Maps, Cognitive Tools.

\section{Introdução}

A nova era das tecnologias da informação e do capital intelectual exige uma mudança profunda na organização, nas teorias curriculares e metodologias de ensino das escolas (Scardamalia, Bereiter, 2000). Apesar das grandes transformações das questões curriculares, observa-se nitidamente no ensino um enquadramento da prática docente a modelos centralizados de ensino, reduzindo-o a uma prescrição do currículo e na execução de programas de instruções codificadas em livros didáticos e em tecnologias instrucionais. Essa visão tecnicista de currículo tem contribuído, por longos anos, para o empobrecimento das ações educativas, restringindo as questões de programa curricular a modelos estáticos de ensino, com características homogêneas, unidimensionais, normativas e seqüenciais (Henriques, 1998).

Além disso, nota-se nas escolas (latino-americanas) escassos recursos didáticos e a inexistência de laboratórios para o ensino de ciências, como exemplo pode-se citar a falta de laboratórios de Física no Ensino Médio. Desse modo, é necessário procurar soluções pedagógico-didáticas consoantes com as necessidades da sociedade contemporânea e que contribuam para a diminuição dos problemas ensinoaprendizagem na educação formal.

Uma proposta de solução seria a aplicação dos recursos computacionais existentes para o desenvolvimento de softwares de apoio ao ensino de Física. Inúmeros estudos relatam a criação de softwares educacionais que simulam fenômenos difíceis de

1 "Bolsista do CNPq - Brasil"

V. 6 № 1, Julho, 2008 
serem realizados em sala de aula. Entretanto, observa-se que muitas dessas tecnologias de ensino não são fundamentadas em modelos cognitivos e pedagógicos, e, portanto, não estimulam processos ativos de aprendizagem (Good, Berger, 1998).

Dessa forma, este projeto se justifica ao agregar um grupo multidisciplinar (Engenharia Elétrica, Educação, Física e Artes) para desenvolver um sistema computacional de apoio ao ensino de Física, fundamentado em modelos pedagógicos e cognitivos contemporâneos.

Para tanto, foi desenvolvido um desenho pedagógico que tem como objetivo estimular à aprendizagem ativa, intencional, reflexiva e significativa dos aprendizes. Com o intuito de eliminar a organização e distribuição linear e unidimensional dos conteúdos foram elaborados um conjunto de organizadores gráficos e mapas conceituais para proporcionar ao aluno uma visualização do todo e das partes entre os conceitos, bem como, a possibilidade de gerenciar a aprendizagem significativa.

Em relação aos aspectos tecnológicos, utilizou-se Realidade Virtual (RV) não imersiva, por meio do desenvolvimento de ambientes virtuais com uso da linguagem VRML (Virtual Reality Modeling Language) e JavaScript, visando criar para o usuário a possibilidade de interagir com ambientes virtuais atrativos, algumas vezes realísticos, que facilitam a aprendizagem significativa de conceitos de Física do Ensino Médio. A opção por técnicas de RV está relacionada com a grande demanda do uso destas técnicas, com o aprendizado e treinamento nas mais diversas áreas de conhecimento tais como Educação, Medicina etc. (Encarnação, 1994; Andrade et al., 1998).

As seções seguintes destacam aspectos relevantes para criação deste trabalho, ressaltando detalhes do desenvolvimento dos ambientes virtuais e mostrando alguns resultados de implementação.

\section{Desenho Pedagógico}

O desenho pedagógico deste software, para a aprendizagem de Física no Ensino Médio, fundamentou-se na análise de desenho pedagógico de Lowyck (2002), nos princípios construtivistas para o desenho de sistemas instrucionais de Lebow (1995), nos modelos de aprendizagem significativa de Ausubel (1984), nos modelos de ensino de ciências de Novak, Mintzes e Wandersee (1998) e nas pesquisas sobre ferramentas cognitivas e tecnologias da informação de Jonassen, Kommers e Mayes (1992).

Elaborou-se três desenhos para os ambientes de aprendizagem. O primeiro ambiente consiste em um sistema de organização e distribuição dos conteúdos por meio de organizadores gráficos conceituais (sistema em árvores). Os organizadores gráficos são excelentes ferramentas cognitivas para a organização e distribuição dos conteúdos da grade curricular, pois eliminam a estrutura modular, fragmentada, linear e unidimensional dos modelos tradicionais de ensino. Dessa maneira, servem como guia para o ensino dos conteúdos de forma multidimensional e interdisciplinar, como também fornecem uma visão entre o todo e as partes dos conceitos a serem estudados. Além disso, contribuem para o gerenciamento da informação e para a aprendizagem significativa porque possibilitam a identificação dos conceitos fundamentais e os conceitos a priori necessários para compreender a nova informação.

O segundo ambiente de aprendizagem foi desenhado para o aluno interagir em sistemas de simulações de fenômenos físicos, em RV, contextualizados com a realidade do aluno. Estes ambientes possuem cenários com diversos objetos de aprendizagem que têm como intuito estimular a ação intencional, a reflexão e a aprendizagem significativa dos aprendizes. As simulações em realidade virtual oferecem a possibilidade de 
exploração de um domínio conceitual, número de objetos que podem ser manipulados, detalhes e a fidelidade das simulações. O processo psicológico que torna ativa a imersão da RV é muito semelhante ao modo que as pessoas adquirem conhecimento, isto é, por meio da interação com objetos e eventos no mundo real (Winn, 1993). Dessa forma, a RV permite que os conceitos científicos possam ser aprendidos de forma concreta, esta característica é consoante com os métodos construtivistas de ensino que estabelecem a necessidade da aprendizagem pela experiência, neste caso, a experiência com objetos virtuais que podem ser observados, explorados e descobertos. Sendo assim, o processo de ensino se desloca do processo tradicional de transmissão da informação para um processo de estímulo a aprendizagem ativa e significativa.

O terceiro ambiente é um sistema tutorial para possibilitar o aluno a gerenciar a aprendizagem significativa e compreender os conceitos envolvidos nos sistemas de simulações dos fenômenos físicos. O sistema tutorial foi desenvolvido por meio de mapas conceituais referentes aos fenômenos físicos das simulações. Os mapas conceituais são ferramentas cognitivas que apresentam uma estrutura formada por um sistema de redes conceituais que se organizam e se distribuem por diferenciações progressivas e reconciliações integrativas, em um modelo que se relaciona por ligações semânticas (ligações proposionais entre os conceitos). As diferenciações progressivas se estruturam de forma que os conceitos superordenados (âncoras para a compreensão da informação) estejam no topo e depois se especificam em conceitos subordinados em diferentes níveis de inclusão. As reconciliações integrativas evidenciam as possíveis relações existentes entre as diversas idéias que estão sendo trabalhadas, de modo a facilitar e a catalisar a criação destas ligações na estrutura cognitiva do aprendiz. Estas ferramentas cognitivas podem ser usadas para gerenciar a aprendizagem, identificando os conceitos fundamentais e os conceitos a priori necessários para compreender a nova informação. Além disso, as pesquisas indicam que a estratégia de formar um todo integrado para os conteúdos a serem ensinados ajuda os alunos a entender o material, clarificar as relações entre os conceitos e diminuir o tempo gasto para os estudantes em memorizar o conteúdo (Jonassen, Peck, Wilson, 1999).

Melhores detalhes sobre o desenho pedagógico dos ambientes de aprendizagem serão expostos na seção sobre desenvolvimento do sistema.

\section{Trabalhos Relacionados}

Com o objetivo de avaliar as potencialidades dos softwares desenvolvidos para o ensino de Física, direcionados ao nível médio, foram analisados alguns trabalhos existentes, procurando ressaltar seus processos de interações, suas estruturas pedagógicas e suas principais características.

\subsection{WebTop}

Com o intuito de ensinar Óptica Geométrica, a Universidade do estado do Mississipi criou a ferramenta WebTOP disponível em URL: http://webtop.msstate.edu/, totalmente desenvolvida em VRML e Java. O sistema é composto por módulos e submódulos que usam simulações computacionais interativas, animadas e em 3D para apresentação de fenômenos físicos. Os sub-módulos possuem acesso para diferentes áreas da Óptica, tais como: Fresnel Diffraction, Fraunhofer Diffraction and Polarization. As principais características observadas neste sistema são: $1^{a}$ ) Desenvolvimento apenas do conteúdo de Óptica. $2^{\mathrm{a}}$ ) $\mathrm{O}$ desenho pedagógico do sistema é direcionado para especialistas da área. $3^{a}$ ) O usuário do WebTop necessita de conhecimentos a priori, bem definidos e claros, necessários para compreensão dos fenômenos ópticos. A 
estrutura pedagógica desta ferramenta não permite ao usuário gerenciar a informação buscando a compreensão dos conceitos e de suas definições relacionadas ao fenômeno, bem como, o acesso aos conceitos necessários para compreender este fenômeno.

\subsection{FisicaNet}

O sistema FisicaNet disponível em URL: http://www.terra.com.br/fisicanet/simulacoes/ possui um conjunto de simulações, no formato applet Java, de diferentes conteúdos de Física do Ensino Médio (Mecânica, Movimentos Ondulatórios, Óptica, etc). As principais características do sistema são: $1^{\text {a }}$ ) As simulações não são modeladas de forma realística, a grande maioria está em apenas duas dimensões (2D), fato que prejudica a associação do experimento com o conhecimento do usuário sobre o real, defasando consideravelmente o seu aprendizado. $2^{a}$ ) Não é possível observar uma estrutura pedagógica no FisicaNet. $3^{a}$ ) As simulações estão dispostas de forma desconexa, existindo apenas textos explicativos, no formato HTML, sobre a simulação em que está sendo executada. $4^{a}$ ) Observa-se que o FisicaNet utiliza fortemente símbolos que não se relacionam com conceitos e suas definições, como também o usuário não tem acesso a notações que representam o fenômeno.

\subsection{LVEF - Laboratório Virtual de Experiências de Física}

O LVEF (Cardoso, 2002) consiste em um sistema de experimentos de Física que permite ao usuário criar seu próprio experimento, utilizando objetos dos ambientes virtuais em VRML e JavaScript. Para tal fim, o sistema utiliza applets Java associados a ambientes virtuais - inicialmente sem elementos - desenvolvidos em VRML. As principais características do sistema são: $1^{a}$ ) O LVEF é um sistema próprio para o ensino direcionado porque não possui uma estrutura pedagógica que permite aos alunos, sem intervenção de professores, a construção de seu próprio conhecimento. $2^{\mathrm{a}}$ ) $\mathrm{O}$ cenário virtual possui poucos objetos em sua biblioteca. $3^{a}$ ) O LVEF simula apenas experimentos virtuais referentes à Mecânica Clássica.

Após analisar estes trabalhos e observar suas limitações a equipe multidisciplinar propôs uma arquitetura para o sistema procurando criar um software que abranja toda a grade curricular do ensino médio, utilizado uma interface em $3 \mathrm{~d}$ e realística, e oferecendo ao usuário uma estrutura pedagógica que dê suporte a sua aprendizagem

\section{Arquitetura do Sistema}

O sistema proposto possui uma interface simples, permitindo a professores e alunos a manipulação fácil dos ambientes virtuais. Não há necessidade de conhecimentos técnicos relacionados à Informática e/ou sobre os conceitos de Física, porque foram criadas estratégias de navegação que orientam os aprendizes no processo de interação nos ambientes de aprendizagem.

A primeira estratégia é a criação de um conjunto de organizadores gráficos conceituais da grade curricular do ensino de Física, para facilitar o processo de navegação conceitual e estimular a aprendizagem significativa. A segunda estratégia é criar o acesso às experiências virtuais de fenômenos físicos por meio dos nós conceituais dos organizadores gráficos. A terceira estratégia é o acesso aos mapas conceituais tutoriais nos ambientes de simulações de experiências virtuais.

Os Ambientes Virtuais do sistema são construídos com a linguagem VRML e JavaScript, o que contribui para a criação de arquivos pequenos, que são compartilhados via Web a um baixo custo de transmissão e armazenamento. A visualização dos 
Ambientes Virtuais é realizada através de um plug-in, como o Cortona, que é executado sob supervisão do navegador da Internet. O usuário pode acessar a GUI (Interface Gráfica do Usuário) do sistema, que oferece suporte a todos processos de criação e visualização dos resultados dos experimentos, presencialmente ou a distância, via Internet. A Figura 1 mostra o diagrama básico da arquitetura do sistema.

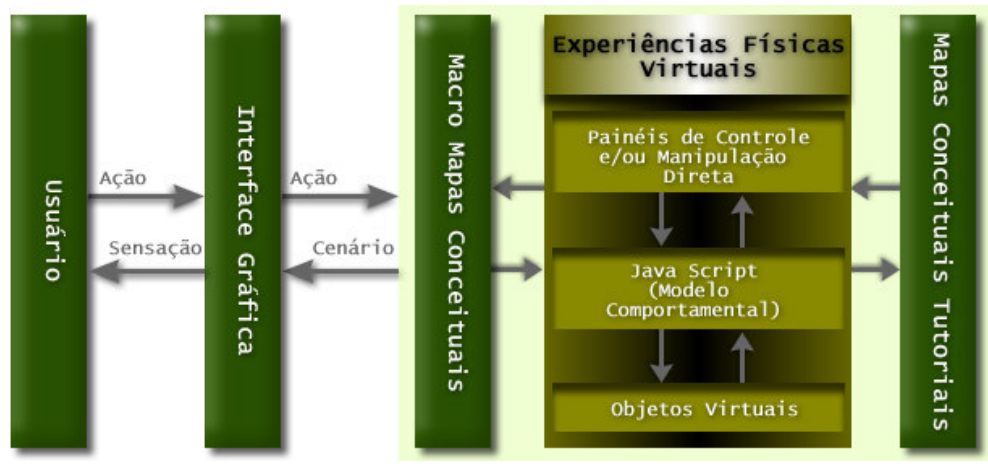

Figura 1. Arquitetura do Sistema.

Os ambientes virtuais dos experimentos são, em grande maioria, modelados de acordo com o mundo real, facilitando deste modo uma melhor associação do conteúdo com o experimento. Um objeto de aprendizagem, padronizado no sistema, de suma importância é o Painel de Controle com variáveis que interferem no fenômeno da experiência física (velocidades, ângulos, acelerações, forças, etc.). Esse painel foi desenvolvido para que o usuário interaja e compreenda as simulações, alterando parâmetros e visualizando os resultados imediatos dessas alterações por meio da mudança de comportamento dos objetos virtuais dispostos em cada ambiente.

\section{Detalhes de Desenvolvimento}

Inicialmente, construiu-se o conjunto de organizadores gráficos conceituais em três dimensões, usando a linguagem VRML. Este modelo teve como objetivo eliminar a organização linear e unidimensional dos conteúdos curriculares formados pelos modelos tradicionais de ensino, e criar um sistema multidimensional e interdisciplinar para a organização e distribuição dos conceitos científicos. Foram utilizados organizadores gráficos para grade curricular porque os mapas conceituais podem confundir visualmente os usuários com a sua estrutura de reconciliações integrativas e estruturas semânticas.

O uso de VRML para organização e distribuição dos conceitos teve como objetivo facilitar a visualização rápida de profundidade do todo da rede conceitual, fato que diminui o potencial dos sistemas bidimensionais que necessitam de navegação por meio de sub-mapas para a visualização da rede conceitual. Ao colocar o mouse sobre cada conceito o usuário pode visualizar os conceitos que virão, se a progressão ocorrer. Deste modo, o estudante pode investigar minuciosamente os conceitos e verificar se possui conhecimento a priori necessário (Subsunçores) para assimilar a nova informação. Se optar em progredir ocorre uma troca para um novo ambiente virtual, que contém conceitos que o usuário já visualizou anteriormente. Os ambientes virtuais apresentam um sistema de redes conceituais com poucos nós na interface, com objetivo de atrair a imagem visual e focalizar a atenção. O usuário pode também acessar as definições conceituais de cada nó. Esse procedimento possibilita o gerenciamento da aprendizagem significativa dos conceitos envolvidos nos fenômenos físicos. O usuário pode colocar o mouse sobre o nó conceitual "Mecânica", por exemplo, e visualizar os 
conceitos que virão, conforme demonstrado na Figura 2. Tal figura também exemplifica a possibilidade do usuário em verificar a definição de cinemática e depois disto fechá-la, evitando confusão visual no ambiente virtual.

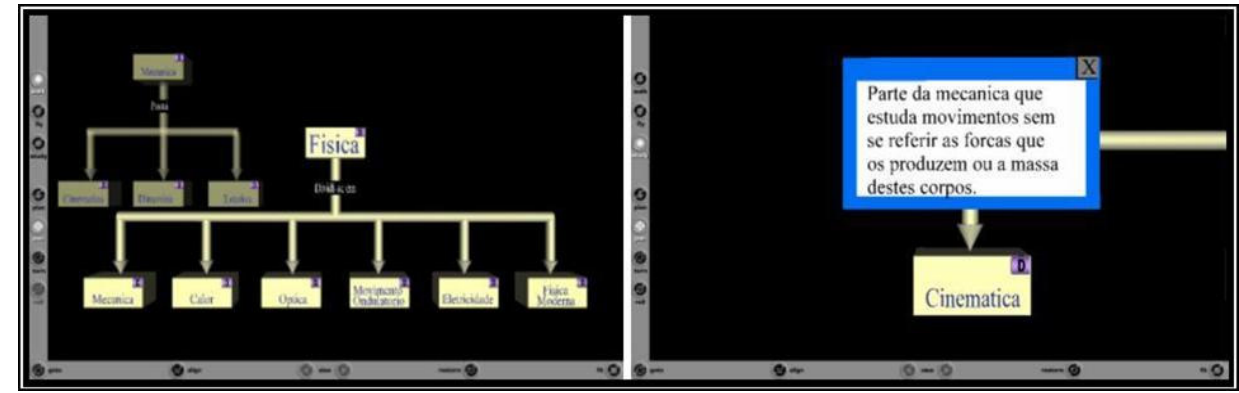

Figura 2. Conceitos após a Progressão / Conceitos e Definições.

Os conceitos mais específicos apresentam ambientes de aprendizagem que possibilitam a interação do aprendiz com simulações de fenômenos físicos em RV, envolvendo toda a rede conceitual de um determinado fenômeno. A Figura 3 mostra que se o usuário quiser requisitar o conceito de Lançamento Oblíquo, ele é transferido para um novo ambiente virtual que é interativo, permitindo-o a observação, exploração e descoberta de fenômenos físicos; podendo inserir e receber dados do ambiente virtual.

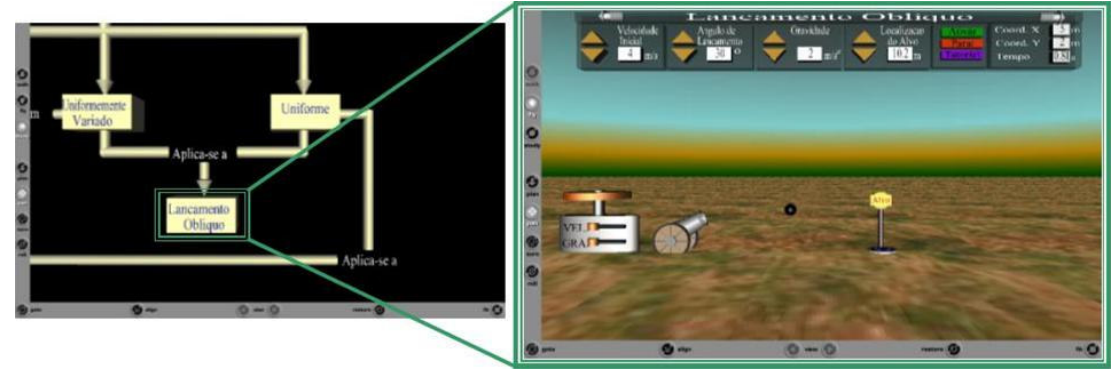

Figura 3. Conceitos - Experiência Lançamento Oblíquo.

Nos ambientes de Experiências Físicas Virtuais, a primeira ação do usuário é inserir os dados nos painéis de controle e/ou manipular diretamente os objetos virtuais. Por exemplo, a experiência de Lançamento Oblíquo (Figura 3) permite duas opções ao usuário: $1^{\mathrm{a}}$ ) pode-se inserir o ângulo de lançamento do canhão através do Painel de Controle, desta forma os dados de entrada são valores inteiros que aumentam ou diminuem de uma quantidade pré-estipulada; $2^{a}$ ) pode-se rotacionar o canhão com o mouse, entrando com qualquer valor desejado seja real ou inteiro.

Após a entrada de dados, o usuário ativa o experimento e os dados são transmitidos para um JavaScript, que os processa de acordo com as equações matemáticas físicas do fenômeno em questão. O JavaScript transmite aos objetos virtuais os cálculos das equações físicas do experimento, estes por sua vez, realizam uma mudança de comportamento (translação, rotação, aumentam ou diminuem de tamanho, etc) no Ambiente Virtual de acordo com estes cálculos. Os resultados do experimento são transmitidos, através do JavaScript, para o Painel de Controle da experiência, onde o usuário pode visualizar de forma instantânea tais resultados.

Em algumas experiências a interação do usuário com o Ambiente Virtual é maior, como no experimento do Movimento Retilíneo Uniformemente Variado (MRUV) que apresenta um cenário contendo alguns quarteirões com sinaleiros e uma caminhonete. $\mathrm{O}$ objetivo do usuário nesta simulação é passar por todos sinaleiros antes 
que fechem e sem ultrapassar o limite de velocidade, podendo acelerar ou frear o automóvel a qualquer momento do experimento. Caso não cumpra seu objetivo, ele é multado a cada infração.

O usuário possui a opção de realizar a experiência de fora da caminhoneta, de forma externa, onde usuário utiliza um ponto de visão que acompanha a caminhoneta durante o experimento, Figura 4 (a), ou de forma aérea visualizando o cenário virtual de cima para baixo como um todo, Figura 4 (b), em ambas as opções o usuário deve utilizar o Painel de Controle para inserir e visualizar os dados de entrada e saída. Executando o experimento de forma aérea, pode-se visualizar um rastro deixado pela caminhonete que muda de cor dependendo das ações do usuário (acelerar, frear ou apenas não executar nenhuma ação). Esse procedimento do cenário virtual oferecer rastro tem como objetivo servir de suporte para análises gráficas do usuário.

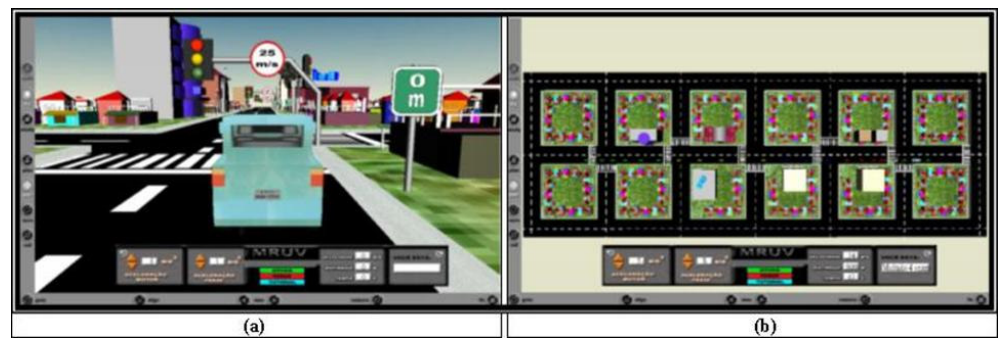

Figura 4. MRUV - Câmeras Externa / Câmera Aérea.

O usuário pode ainda realizar o experimento dentro da caminhoneta utilizando seu painel para visualizar os resultados da experiência, como ilustra a Figura 5.

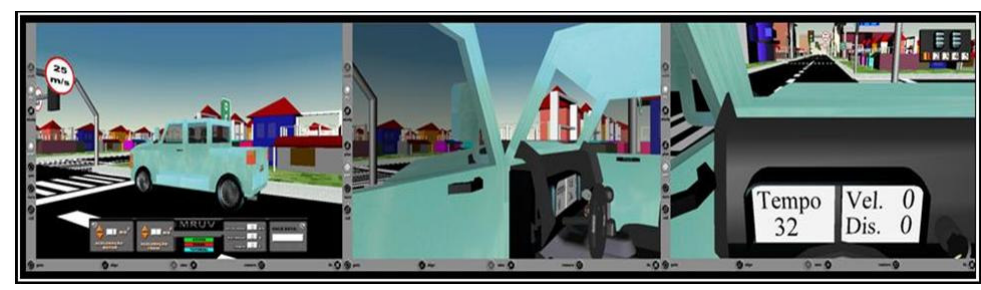

Figura 5. MRUV - Câmera Interna.

Existem também três câmeras localizadas em pontos pré-definidos no cenário virtual que permanecem fixas e acompanham o movimento da caminhonete durante seu trajeto. Esse artifício orienta a reflexão do usuário sobre os conceitos de referencial e movimento relativo.

Quando o usuário está realizando um experimento, ele consegue acessar, a qualquer momento, o mapa conceitual tutorial referente aos conceitos envolvidos naquele fenômeno. Este tutorial tem como objetivo facilitar a assimilação dos conceitos e suas relações de um determinado fenômeno físico, bem como, estimular a memória compreensiva em longo prazo.

Para ter acesso a este mapa conceitual basta utilizar a opção 'Tutorial', presente no Painel de Controle de cada experiência. Na construção destes mapas conceituais utilizou-se a linguagem HTML porque apresenta uma fácil visualização das reconciliações integrativas e podem ser inseridos hipertextos para explicações mais aprofundadas. Esses procedimentos de acessos aos hiperlinks nos mapas conceituais têm como objetivo servir de suporte para o gerenciamento da aprendizagem, visto que os alunos podem assimilar novas informações e perceber os conceitos que não compreendem. 
Finalmente, no desenvolvimento deste software foram construídas vinte e seis experiências físicas em VRML, contextualizadas e organizadas por organizadores gráficos e mapas conceituais, abrangendo a grade curricular de Física do Ensino Médio.

\section{Avaliação}

Com base em preceitos da ISONORM 9241-10 Usability e de acordo com o Checklist do PROINFO foi elaborado um questionário de avaliação para o sistema. A ISONORM 9241-10 Usability é um conjunto de normas que permite avaliar a capacidade de um sistema interativo oferecer a seu usuário a possibilidade de realizar tarefas de maneira eficaz e agradável (Prugmper, 1999). Já o Checklist do PROINFO, disponível em URL: http://www.uel.br/seed/ nte/analisedesoftwares.html, estabelece critérios para avaliação especificamente de softwares educacionais.

O questionário foi aplicado a trinta e quatro alunos do Ensino Médio de diferentes escolas e avaliou os ambientes de aprendizagem do sistema separadamente, os organizadores gráficos, as experiências físicas virtuais e os mapas conceituais. Tal metodologia fez-se necessária, pois cada ambiente possui desenhos pedagógicos e interfaces diferentes. Foram abordas ainda algumas questões sobre o contato dos usuários com computadores e laboratórios de ensino de Física, as quais identificaram que $50 \%$ dos alunos usavam o computador com muita freqüência e que apenas $32 \%$ dos entrevistados haviam desenvolvido experimentos de Física em laboratórios durante o Ensino Médio.

De posse dos resultados dos questionários de avaliação foram realizados alguns estudos de Estatística Descritiva e construiu-se gráficos percentuais comparativos baseados em alguns parâmetros específicos da ISONORM 9241-10 Usability. Tais parâmetros são: (a) compatibilidade, que retrata a adequação das funções do sistema para as ações do usuário; (b) códigos/denominações, que refere-se à clareza dos termos utilizados no software; (c) homogeneidade/coerência, que retrata a facilidade de uso do programa; (d) gestão de erros, que refere-se à qualidade da ajuda ao usuário e as informações de uso do software; (e) adaptabilidade, que retrata as diferentes maneiras de se realizar uma tarefa; (f) controle explícito, que refere-se ao controle sobre as atividades do software e a confiança na execução destas. (g) carga de trabalho, retrata o número de passos e funções utilizadas para executar uma determinada tarefa; (h) condução, que refere-se à agilidade de acesso a funções, aspectos gráficos da tela e, o entendimento de ícones. A Figura 6 ilustra o gráfico resultante deste estudo.

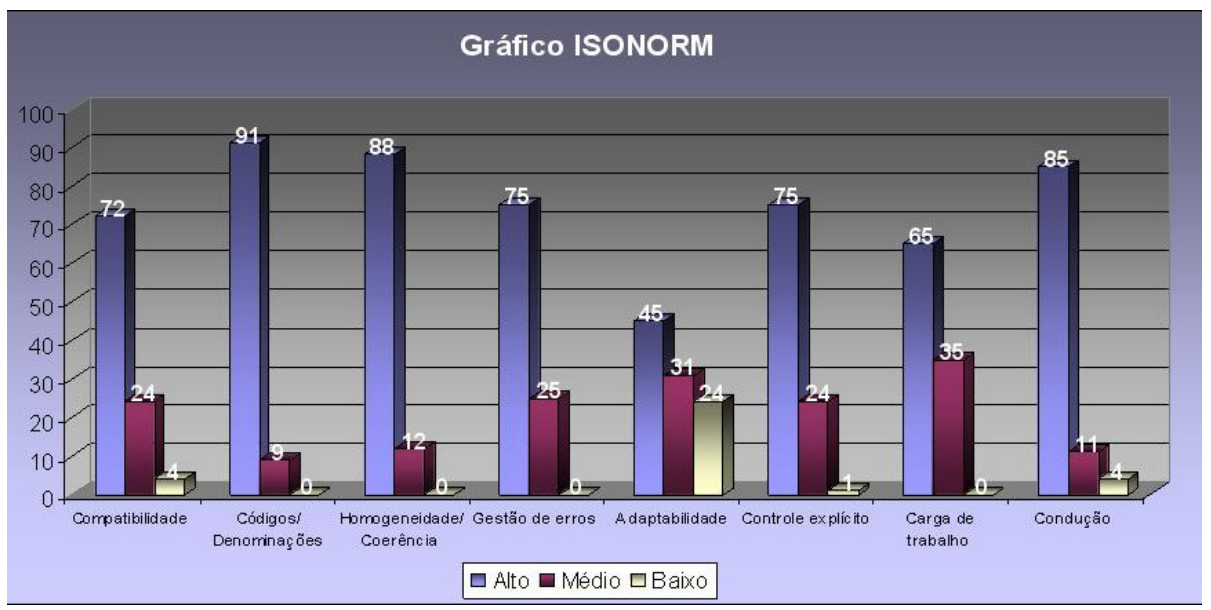

Figura 6. Gráfico baseado em parâmetros da ISONORM. 
A partir destes resultados foi possível chegar a algumas conclusões sobre este trabalho, as quais são discutidas abaixo.

\section{Conclusão}

No decorrer desta pesquisa, verificou-se a existência de diversos softwares voltados para o ensino de Física. No entanto, a maioria não apresenta uma fundamentação pedagógica explícita de seus ambientes, o que conseqüentemente acarreta desvios na eficiência do processo ensino e aprendizagem. Diante disso, este estudo contribui com as pesquisas atuais em tecnologias da informação na educação, ao desenvolver um software para o ensino de Física, fundamentado em desenhos pedagógicos para ambientes computacionais. Apresenta diversos cenários e objetos de aprendizagem em Realidade Virtual, com o objetivo de estimular o gerenciamento da aprendizagem (aprender a aprender), a aprendizagem intencional e significativa de conceitos em Física que possam ser transferidos para outros contextos de aprendizagem.

Pode-se afirmar que o produto final desse software obteve melhoras significativas devido às contribuições da equipe multidisciplinar. A equipe da Educação contribuiu com a arquitetura e os desenhos pedagógicos dos cenários e objetos de aprendizagem dos três ambientes do sistema. A equipe de Física ofereceu um suporte conceitual para o sistema e estratégias para a criação de simulações. A equipe de Artes Virtuais contribui com interfaces ergonômicas para os ambientes virtuais e algumas modelagens de objetos.

A integração do VRML com o JavaScript foi de suma importância na implementação. A linguagem VRML foi apropriada para modelar os mundos virtuais, porém para incluir interações, animações e realizar cálculos foi preciso utilizar uma linguagem de programação específica que contemplasse comandos (JavaScript). O uso de tal técnica garantiu a possibilidade do sistema desenvolvido ser acessado via Internet, sem custo de aquisição de softwares e hardwares adicionais.

De acordo com a avaliação dos usuários, o sistema apresenta-se como uma das principais contribuições para uso de processos pedagógicos integrados a técnicas de RV. A adoção de princípios e técnicas pedagógicas e as técnicas de RV exploradas indicaram uma maior motivação do usuário para o gerenciamento da informação e construção de seu conhecimento de forma mais intuitiva que outros tutoriais encontrados na literatura e na Internet.

Verificou-se que o SEFIRV foi bem aceito e de fácil usabilidade. Além disso, constatou-se também que os alunos não têm acesso freqüente ao laboratório de ensino de Física; pelo contrário, utilizam constantemente o computador. Diante desses fatos, indica-se que esse software pode oferecer suporte ao ensino de Física, tornando-se uma ferramenta de apoio às escolas e aos alunos que não têm acesso a laboratórios de Física instalados. A avaliação demonstra que as estratégias de representação de fenômenos físicos, elaboradas para o desenvolvimento dos ambientes virtuais, foram bem avaliadas pelos usuários, sendo consideradas úteis para a aprendizagem em Física.

Os usuários consideraram que os organizadores gráficos e os mapas conceituais ajudaram no seu aprendizado, devido a sua organização, distribuição e as relações entre os conceitos. No entanto, não foi possível avaliar as contribuições destas ferramentas cognitivas para a memorização, a longo prazo, dos conceitos.

O desenvolvimento deste trabalho demonstrou o grande potencial que a tecnologia de Realidade Virtual oferece à área educacional, principalmente se esta tecnologia estiver aliada a processos pedagógicos. 
E finalmente, é possível verificar que esta pesquisa contribuiu para melhoria do entendimento sobre o uso das técnicas de Realidade Virtual não-imersiva e ferramentas cognitivas (mapas conceituais e organizadores gráficos) como ferramentas auxiliares ao processo de ensino e aprendizagem.

\section{Referências}

SCARDAMALIA, M.; BEREITER, C. Schools as knowledge Building Organizations. Ontario Institute for Studies in Education of the University of Toronto, 2000 .

HENRIQUES, M. S. O Pensamento Complexo e a Construção de um Currículo Não-Linear. Reunião Anual da ANPED. 1998.

GOOD, R.; BERGER, C. The Computer as Powerful tool for Understanding Science. In. Mintzes, Wandersee and Novak, ed. Teaching Science for Understanding: A Human Constructivist View. Academic Press, San Diego, 1998.

ENCARNAÇÃO, J. European Activities in Virtual Reality. In: IEEE Computer Graphics and Applications, página 73, 1994.

ANDRADE, F. A.; WAZLAWICK, S. R.; CRUZ, D. M. Realidade Virtual na Escola: Um Panorama. XVIII Congresso Nacional da Sociedade Brasileira de Computação SBC, 18. Belo Horizonte, Brasil, páginas. 604 - 613, 1998.

LOWYCK, J. Pedagogical Design. In: Adelsberger, Collis and Pawlowski ed. Handbokk on Information Technologies for Education and Training. International. Handbooks on information Systems. Springer Verlag, Berlin, 2002.

LEBOW, D. Constructivist Values for Instructional Systems Design: Five Principles Toward a New Mindset. In Seels (ed.) Instructional Design Fundamentals: A Reconsideration Educational Technology Publications, Englewood Cliffs, New Jersey, 1995.

AUSUBEL, D.; NOVAK, N.; HANASSEN, D. Psicologia Educacional. Interamericana. Rio de Janeiro, Brasil, 1984.

NOVAK, J.; MINTZES, J.; WANDERSEE, J. Teaching Science for Understanding: A Human Constructivist View. Academic Press, San Diego., 1998.

JONASSEN, D.; KOMMERS, P.; MAYES, J. Cognitive Tools for Learning. SpringVerlag, Berlin, 1992.

WINN, W. A Conceptual Basis for Educational Applications of Virtual Reality. Human Interface Technology Laboratory, Washington Technology Center, 1993.

JONASSEN, D.; PECK, K.; WILSON, B. Learning with Technology: A Constructivist Perspective. Prentice Hall, Ohio, 1999.

CARDOSO, A. Uma Arquitetura para Elaboração de Experimentos Virtuais Interativos suportados por Realidade Virtual Não-imersiva, Tese de Doutorado, USP, 2002.

PRUGMPER, P. Test it: ISONORM 9241/10. In: H.-J. BULLINGER \& J. ZIEGLER Eds. Proceedings of HCI International, Munich, 22-27 August Mahwah 1999, NJ: Lawrence Erlbaum, 1999. 